\title{
Top Quark Physics at CMS
}

\section{Andreas MEYER*}

DESY

E-mail: andreas.meyerdesy.de

Experimental results on top-quark physics obtained at the CMS experiment are reported. The results are based on the data recorded during the years 2011 and 2012 at centre-of-mass energies of $7 \mathrm{TeV}$ and $8 \mathrm{TeV}$. CMS delivered a wealth of top-quark measurements ranging from inclusive and differential cross sections for top-quark pair and single top-quark production through the analysis of top-quark properties in production and decay, such as spin correlations, charge asymmetries and top-quark mass measurements, to searches for anomalous couplings. The measurements provide crucial information on the validity of approximations in QCD calculations as well as important constraints of fundamental standard model parameters and stringent limits on new physics searches.

XIIth International Conference on Heavy Quarks \& Leptons 2014

25-29 August 2014

Schloss Waldthausen, Mainz, Germany

${ }^{*}$ Speaker. 


\section{Introduction}

The top quark is by far the heaviest known elementary particle. Due to its large mass the top quark decays within $5 \cdot 10^{-25} \mathrm{~s}$, before hadronisation, and thus gives direct access to its properties such as spin and charge. With its large mass, the top quark is expected to couple strongly to the Higgs boson and thus to play a crucial role in electroweak loop corrections. Not least, top-quark measurements provide important input to QCD calculations. The measurements help discriminate between different perturbative approaches, and have the potential to constrain QCD parameters. In the standard model, due to the largeness of the CKM-matrix element $V_{t b}$, top quarks decay almost exclusively to a W-boson and b-quark. The decay channel of the W-boson into leptons or quarks is then generally used to distinguish different top-quark decay channels.

At the CERN Large Hadron Collider (LHC), the main production mechanism of top quarks is top-quark pair production, via the strong interaction. The top quark can also be produced via the electro-weak interaction. Between 2010 and 2012, about five million top-quark events were produced in the CMS experiment, and a very large fraction of these events was successfully triggered, recorded and reconstructed. The largest dataset, corresponding to a luminosity of about $20 / \mathrm{fb}$ was recorded in 2012 when the LHC operated at a proton-proton centre-of-mass energy of $8 \mathrm{TeV}$.

The main feature of the CMS experiment is a large magnetic coil that provides a magnetic field of 3.8 Tesla. All detectors, except the muon detection systems are placed within this coil. The inner tracking detectors are entirely based on Silicon pixel and strip detectors [1]. The electromagnetic calorimeter is a Crystal-Calorimeter. Identification of electrons, muons and photons as well as the reconstruction of jets rely on the "particle-flow" algorithm where the information from all subdetectors is combined to optimal response and resolution [2]. The energy scale uncertainty for jets typically varies between 1 and $3 \%$ depending on the transverse momentum of the jets. In events containing top-quark pairs, two jets originate from the hadronization of a $b$ quark. The identification of such jets relies on the tracks and vertices that are displaced from the primary vertex, due to the finite lifetime of B-hadrons [3].

\section{Top-Quark Pair Cross Section Measurements}

Full next-to-next-to-leading order (NNLO) QCD corrections for the inclusive top-quark pair production cross section in proton-proton collisions became available recently [4]. For a $p p$ centreof-mass energy of $8 \mathrm{TeV}$, assuming a top-quark mass of $173.3 \mathrm{GeV}$, the predicted top-quark pair cross section is $245.8_{-8.4}^{+6.2}(\text { scale })_{-6.4}^{+6.2}(P D F)$. The theoretical precision poses a serious challenge to experiments and also allows for precise extraction of QCD parameters, such as the pole mass of the top-quark, as well as the setting of limits on contributions from beyond-the-standard-model physics.

The top-quark pair cross section measurements from CMS [5, 6] are shown in Figure 1, both at 7 and $8 \mathrm{TeV}$. Also shown is the theoretical calculation, repeated here for a top-quark mass of 172.5 $\mathrm{GeV}$. The top-quark pair cross section can also be used to extract the pole mass of the top quark and the value of the strong coupling constant $\alpha_{S}$ [7]. Using the most precise cross section measurement at $7 \mathrm{TeV}$, in the dilepton channel, CMS measured the top-quark pole mass as $176.7_{-2.8}^{+3.0} \mathrm{GeV}$, fixing $\alpha_{S}\left(m_{Z}\right)$ to the value of 0.1151 and using the parton distribution function NNPDF2.3 [8]. 

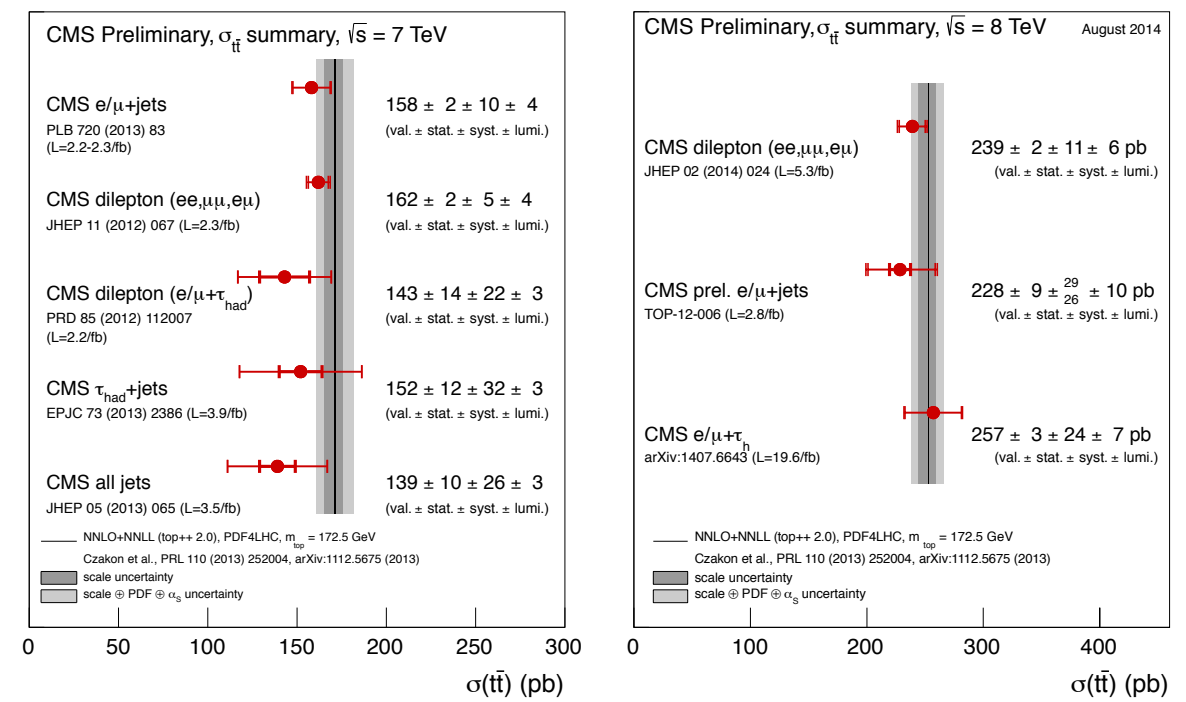

Figure 1: Summary of top-quark pair cross section measurements by the CMS experiment at centre-of-mass energies of $7 \mathrm{TeV}$ (left panel) and $8 \mathrm{TeV}$ (right panel).

Conversely, fixing the pole mass to $173.2 \pm 1.4 \mathrm{GeV}$, a precise value of $\alpha_{S}\left(m_{Z}\right)=0.1151_{-0.0027}^{+0.0028}$ was determined.

Detailed studies of differential cross sections were performed as functions of the various final state particles, of the top quarks and also of global observables such as the scalar sum of the transverse energy in the events. Furthermore, distributions of additional jets in $t \bar{t}$ events were measured. CMS performed these measurements in the $1+$ jets and dilepton channels both for 7 and $8 \mathrm{TeV}$ [9]. Among the many measured distributions, the most striking finding was in the spectrum of the transverse momentum of top quarks. For the top-quark transverse momentum distribution, as well as for the corresponding distributions of the top-quark decay particles, the CMS measurements consistently show a somewhat steeper fall-off of the data than expected by the Monte Carlo simulations which include calculations up to next-to-leading order accuracy in QCD perturbation theory. The discrepancy between data and simulation is presently under investigation. A good description of the data is achieved by calculations to approximate next-to-next-to-leading order. For the time being it constitutes an important source of uncertainty for many analyses. CMS also performed analyses of top-quark pair events with additional identified final-state particles, $t \bar{t}+X$, where $X=b \bar{b}, t \bar{t}, Z, \gamma$ or $\mathrm{W}[10]$. Among these the processes $t \bar{t}+b \bar{b}$ and $t \bar{t}+V(=Z, \gamma, W)$ are important backgrounds to events in which a Higgs boson is produced in association with a topquark pair. In the future, experimental measurements will help reduce the theoretical uncertainties which are currently still large.

\section{Single Top Quark Measurements and $V_{t b}$}

Processes involving the production of one single top quark proceed via the electro-weak interaction. Depending on whether the W-boson is space-like, time-like or real, one distinguishes 

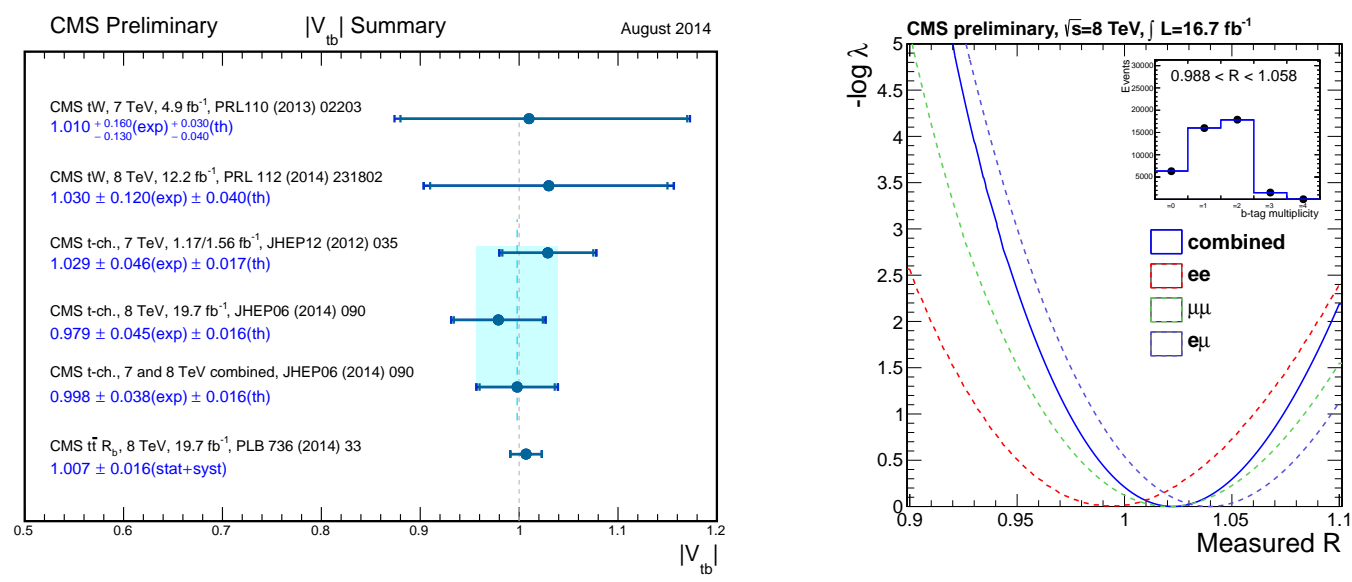

Figure 2: Left: Results for $V_{t b}$ from various measurements of single top-quark production and of $t \bar{t}$ decays. Right: Variations of the likelihood ratio used to extract $R_{B}$ from the data. The variations observed in the combined fit and in the exclusive $e e, \mu \mu$, and $e \mu$ channels are shown separately. The inset shows the inclusive b-tagged jet multiplicity distribution and the fit distribution.

between the $t$-channel, the $s$-channel and the $t W$-channel. In the latter case, a single top quark is produced in association with a $W$ boson in the final state. The expected cross sections for single top-quark production at a centre-of-mass energy of $8 \mathrm{TeV}$, and a top-quark mass of $173 \mathrm{GeV}$ are $87.2_{-1.0-2.2}^{+2.1+2.0} \mathrm{pb}, 22.2 \pm 0.6 \pm 1.4 \mathrm{pb}$ and $5.55 \pm 0.08 \pm 0.21 \mathrm{pb}$ for $t, t W$ and $s$-channel, resp. [11]. CMS measured the cross section of the $t$-channel to be $83.6 \pm 2.3 \pm 7.4 \mathrm{pb}$ at $8 \mathrm{TeV}$ [12], consistent with the SM. The charge ratio $\sigma(t) / \sigma(\bar{t})$ has also been measured and the result is in good agreement within uncertainties with most common parton distribution functions [12]. At the LHC, the measurement of the $t W$-channel has become accessible for the first time. For this process, CMS measures a cross section of $23.4 \pm 5.4 \mathrm{pb}$ at a centre-of-mass energy of $8 \mathrm{TeV}$ [13]. The cross section for the s-channel is very small in comparison and the measurement heavily suffers from backgrounds that dominantly originate from $t \bar{t}$ events. CMS has determined an upper limit on the cross section of $11.5 \mathrm{pb}$ at $95 \%$ C.L. [14].

Single top-quark production measurements also give access to the determination of the modulus of the CKM matrix element $V_{t b}$ which describes the strength of the $W t b$ coupling at the topquark production vertex. Assuming that $\left|V_{t b}\right| \gg\left|V_{t s}\right|,\left|V_{t d}\right|$, the value of $\left|V_{t b}\right|^{2}$ is determined from the ratio of measured and calculated cross sections. Assuming unitarity in addition, i.e. $\left|V_{t b}\right|<1$, lower limits can be determined. The most precise results are summarised in Figure 2 (left).

A significantly more precise determination of $V_{t b}$ can be obtained from the measurement of the ratio $R_{B}=B(t \rightarrow W b) / B(t \rightarrow W q)$ using top-quark pair events. CMS has presented a measurement of $R_{B}$ using a binned-likelihood function of the observed b-tagging multiplicity distributions in events with two, three, or four observed jets in the different dilepton channels [15]. In Figure 2 (right) the variation of the profile likelihood ratio is shown. The fit yields a value $R_{B}=1.014 \pm$ 0.003 (stat.) \pm 0.032 (syst.). Assuming the CKM matrix to be unitary, a lower limit for $V_{t b}$ of 0.975 is set at $95 \%$ confidence level. 

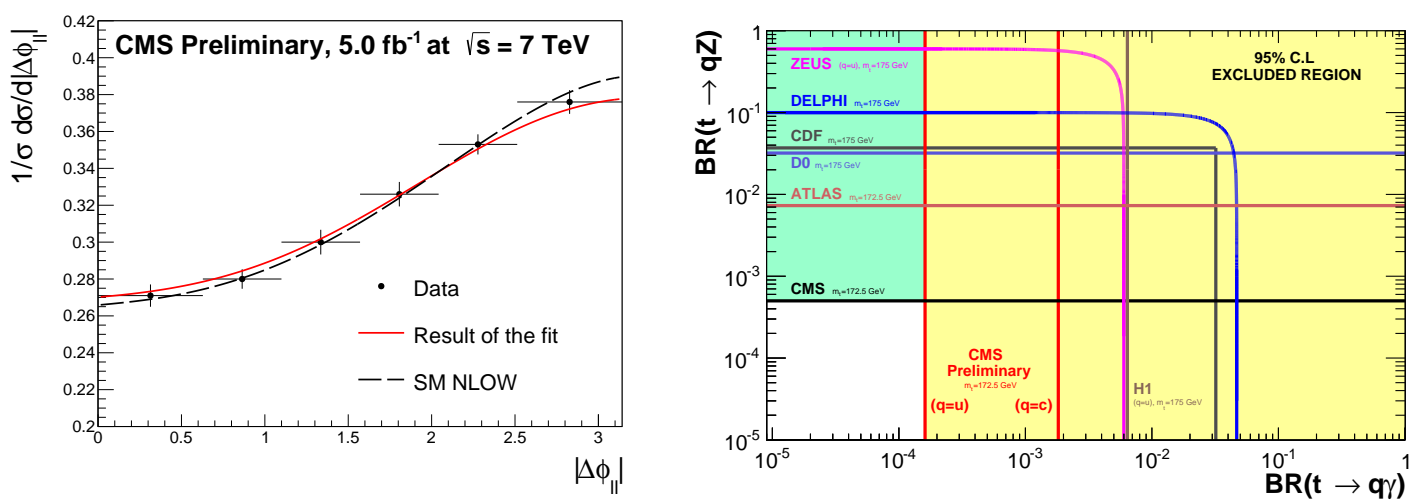

Figure 3: Left: The differential cross section as a function of the opening angle in azimuth between the two final state leptons. The lines show the prediction from the standard model (NLOW) and the result from the fit to the data. Right: The observed 95\% C.L. upper limits on the branching ratios for the processes $t \rightarrow q Z$ and $t \rightarrow q \gamma$ for the DELPHI, ZEUS, H1, DÃ Ÿ, CDF, ATLAS and CMS collaborations.

\section{Properties Measurements}

In the Standard Model, at leading order, top-quark pairs are produced in a symmetric state. The underlying hard scattering processes are $q \bar{q} \rightarrow t \bar{t}$, and gluon-fusion, $g g \rightarrow t \bar{t}$. At NLO, additional diagrams arise which break this charge symmetry, namely quark-gluon scattering, $q g \rightarrow t \bar{t}$, and radiative corrections to quark-antiquark annihilation [16]. At the Tevatron experiments, where the initial state is $p \bar{p}$, experimentally a forward-backward asymmetry can be measured. In contrast, at the LHC, the initial state is symmetric, and a charge asymmetry is induced only from the (momentum) difference of the (valence+sea) quark and (sea) anti-quark distributions in the protons, leading to a difference of absolute rapidities of top quarks and anti-quarks, $\left|y_{t}\right|-\left|y_{\hat{t}}\right|$. CMS has published several measurements, including measurements differential in the kinematics of the $t \bar{t}$ system [17]. All measurements are consistent with the SM expectations within still relatively large uncertainties.

If top quarks are spin-1/2 particles that behave according to Standard Model expectations, then top quarks are unpolarized in $t \bar{t}$ production, and their spins are correlated. This expectation can be tested by measuring the angular distributions of the leptons from the decays of the $\mathrm{W}$ bosons which carry the information on the spin history of the event. In Figure 3 (left), a measurement of the distribution of the azimuthal angle between the two leptons in the laboratory system is displayed together with the prediction from QCD [18]. This experimental result can be used to set limits of new physics processes with anomalous couplings that would lead to changes in the distribution. CMS reports a limit on the real part of the chromo-magnetic dipole moment $\mu_{t}$ of $0.043<\operatorname{Re}\left(\mu_{t}\right)<0.117$ at $95 \%$ C.L. [19].

CMS has also measured the polarization of top quarks in single top-quark production and found consistency with the expectation from the left-handedness of the $W t b$ vertex [20]. The chirality of this vertex is reflected in the helicity of W-bosons in top-quark decays. CMS has performed several measurements in different final states of top-quark pair events and also an event 

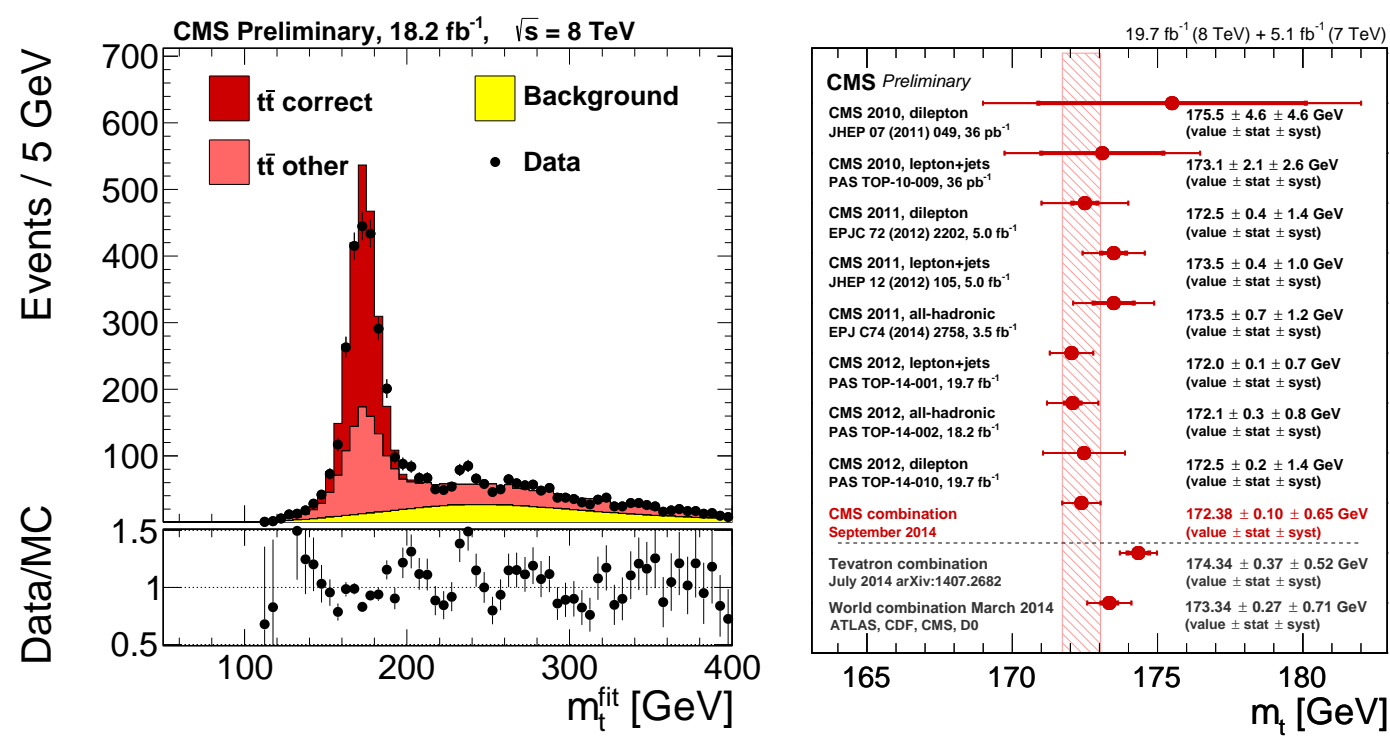

Figure 4: Left: Reconstructed top-quark mass from the kinematic fit to the data sample in the fully hadronic channel. The simulated $t \bar{t}$ signal and the background are normalized to the data. Right: Summary of the eight CMS top-quark mass measurements and their combination.

sample with an enhanced contribution from single top quark events, and found good agreement with the expectation assuming pure V-A coupling according to the standard model [21].

Flavour-Changing Neutral Currents (FCNC) are highly suppressed in the standard model and may only occur via processes beyond tree-level for which the standard model branching ratios are smaller than $10^{-10}$ [22]. The experimental measurement of significantly larger rates would constitute a clear evidence for the presence of new physics. CMS has been performing several searches for FCNC involving top quarks $t \rightarrow X(=\gamma, Z, g, H)+q(=c, u)$ in both production and decay [23]. A summary of the results for the channels $t \rightarrow q Z$ and $t \rightarrow q \gamma$ is given in Figure 3 (right).

\section{Mass Measurements}

The mass of the top quark is a fundamental parameter within the SM and affects predictions of SM observables via radiative corrections. From a theoretical point of view, a meaningful definition of the top-quark mass requires to specify the renormalisation scheme used to define the parameter in the theoretical predictions. Experimentally, the mass of the top quark is generally determined through the reconstruction of the top quark's decay products. The measured mass value is then determined from the simulation by comparing the reconstructed mass values in the data and in the simulation. Experimentally, this approach achieves the greatest precision. However, pole mass and MC mass values can only be identified with each other at an uncertainty of order $1 \mathrm{GeV}$.

Experimentally, CMS has measured the top-quark mass from $t \bar{t}$ events, in the decay channels dilepton, lepton+jets and all-hadronic channels, using data at 7 and $8 \mathrm{TeV}$, and employing a large variety of methods and observables [24, 25]. In Figure 4 (left) the reconstructed top-quark mass 
distribution is shown for the case of the analysis in the fully hadronic channel at a centre-of-mass energy of $8 \mathrm{TeV}$. A tight event selection, requiring at least 6 jets of which two are identified as b-jets, together with a kinematic reconstruction in which the reconstructed mass of the W-boson is fixed to $80.4 \mathrm{GeV}$ and the masses of both top-quark candidates in the event are required to be the same, yields a width of the mass distribution of about $8 \mathrm{GeV}$. The latest CMS combination of the top-quark mass measurements yields the value 172.2 $\pm 0.1 \pm 0.7 \mathrm{GeV}$ [25]. A summary of the CMS measurements and their combination in comparison with results from the Tevatron and the World combination is given in Fig. 4 (right).

Alternative mass measurements are directed towards reducing experimental and/or theoretical uncertainties. The theoretically best-defined measurement is the extraction of the top-quark pole mass from the cross section. The CMS result was already presented in section 2 above [7]. Experimentally, dominant uncertainties arise from the knowledge of the jet energy scale as well as from the modelling of soft non-perturbative effects, such as the underlying event, color-reconnection and multi-parton interactions. CMS has performed a variety of measurements that are designed to complement the central results [26]. These include the measurement of the top-quark mass from the endpoint of the invariant mass distribution and the use of the transverse decay length $L_{x y}$ of the $B$-hadron in top-quark decays. The goal is to reduce the uncertainties further by combination of all measurements.

\section{Conclusions}

The CMS collaboration has produced a wealth of results on top-quark physics within the few first years or data taking at the LHC. The measurements provide important constraints of standard model parameters, such as the top-quark mass and the CKM matrix-element $V_{t b}$, as well as QCD parameters $\alpha_{S}$ and parton density distributions. The standard model calculations are able to describe the data well. The precision of the data increases the modelling of the strong interactions, both w.r.t. higher-order effects as well as soft non-perturbative processes becomes increasingly relevant. While more results from LHC Run 1 are still underway, CMS is preparing for the data taking at yet increased centre-of-mass energy and luminosity. Further insights are expected from more measurements at further improved precision.

\section{References}

[1] CMS Collaboration, JINST 3 (2008) S08004.

[2] CMS Collaboration, CMS-PAS-PFT-09-001.

[3] CMS Collaboration, CMS-PAS-BTV-13-001.

[4] M. Czakon, P. Fiedler, A. Mitov, Phys. Rev. Lett. 110 (2013) 252004 [arXiv:1303.6254].

[5] CMS Collaboration, Phys. Lett. B 720 (2013) 83 [arXiv:1212.6682], Eur. Phys. J. C 73 (2013) 2386 [arXiv:1301.5755], JHEP 11 (2012) 067 [arXiv:1208.2671], Phys. Rev. D 85 (2012) 112007 [arXiv:1203.6810], JHEP 05 (2013) 065 [arXiv:1302.0508].

[6] CMS Collaboration, JHEP 02 (2014) 024 [arXiv:1312.7582], Phys. Lett. B 739 (2014) 23 [arXiv:1407.6643], CMS-PAS-TOP-12-006. 
[7] CMS Collaboration, Phys. Lett. B 728 (2013) 496 [arXiv:1307.1907].

[8] R.D. Ball et al., Nucl. Phys. B 855, 153 (2012) [arXiv:1107.2652].

[9] CMS Collaboration, EPJC 73 (2013) 2339 [arXiv:1211.2220], Eur. Phys. J. C 74 (2014) 3014 [arXiv:1404.3171], CMS-PAS-TOP-12-019, CMS-PAS-TOP-12-041, CMS-PAS-TOP-12-042, CMS-PAS-TOP-12-027, CMS-PAS-TOP-12-028.

[10] CMS Collaboration, Submitted to Phys. Lett. B [arXiv:1411.5621], Submitted to JHEP [arXiv:1409.7339].

[11] N. Kidonakis: arXiv:1311.0283

[12] CMS Collaboration, JHEP 06 (2014) 090 [arXiv:1403.7366].

[13] CMS Collaboration, Phys. Rev. Lett. 112 (2014) 231802 [arXiv:1401.2942].

[14] CMS Collaboration, CMS-PAS-TOP-13-009.

[15] CMS Collaboration, Phys. Lett. B 736 (2014) 33 [arXiv:1404.2292]

[16] J. Kühn, G. Rodrigo: hep-ph/9802268, hep-ph/9807420.

[17] CMS Collaboration, Phys. Lett. B 717 (2012) 129 [arXiv:1207.0065], JHEP 04 (2014) 191 [arXiv:1402.3803], CMS-PAS-TOP-12-033.

[18] CMS Collaboration, Phys. Rev. Lett. 112 (2004) 182001 [arXiv:1311.3924].

[19] CMS Collaboration, CMS-PAS-TOP-14-005.

[20] CMS Collaboration, CMS-PAS-TOP-13-001.

[21] CMS Collaboration, Submitted to JHEP [arXiv:1410.1154], JHEP 10 (2013) 167 [arXiv:1308.3879], CMS-PAS-TOP-12-015.

[22] J. A. Aguilar-Saavedra, Acta Phys.Polon.B35:2695-2710,2004 [hep-ph/0409342].

[23] CMS Collaboration, Phys. Rev. Lett. 112 (2014) 171802 [arXiv:1312.4194], Phys. Lett. B 718 (2013) 1252 [arXiv:1208.0957], CMS-PAS-TOP-14-007, CMS-PAS-TOP-14-003, CMS-PAS-TOP-12-021.

[24] CMS Collaboration, JHEP 12 (2012) 105 [arXiv:1209.2319], Eur. Phys. J. C 72 (2012) 2202 [arXiv:1209.2393], Eur. Phys. J. C 74 (2014) 2758 [arXiv:1307.4617], CMS-PAS-TOP-14-001, CMS-PAS-TOP-14-002, CMS-PAS-TOP-14-010.

[25] CMS Collaboration, CMS-PAS-TOP-14-015.

[26] CMS Collaboration, CMS-PAS-TOP-12-029, Eur. Phys. J. C 73 (2013) 2494 [arXiv:1304.5783], JHEP 06 (2012) 109 [arXiv:1204.2807], CMS-PAS-TOP-12-031, CMS-PAS-TOP-13-007, CMS-PAS-TOP-12-030. 